\title{
Self-Trapped Exciton Defects in a Charge Density Wave: Electronic Excitations of $\mathrm{BaBiO}_{3}$
}

\author{
Philip B. Allen and Ilka B. Bischofs \\ Department of Physics and Astronomy, State University of New York, Stony Brook, New York 11794-3800
}

(October 26, 2018)

In the previous paper, it was shown that holes doped into $\mathrm{BaBiO}_{3}$ self-trap as small polarons and bipolarons. These point defects are energetically favorable partly because they undo locally the strain in the charge-density-wave (Peierls insulator) ground state. In this paper the neutral excitations of the same model are discussed. The lowest electronic excitation is predicted to be a self-trapped exciton, consisting of an electron and a hole located on adjacent $\mathrm{Bi}$ atoms. This excitation has been seen experimentally (but not identified as such) via the Urbach tail in optical absorption, and the multi-phonon spectrum of the "breathing mode" seen in Raman scattering. These two phenomena occur because of the Franck-Condon effect associated with oxygen displacement in the excited state.

71.38+i, 71.45.Lr

\section{INTRODUCTION}

The previous paper on $\mathrm{BaBiO}_{3}$ [1] presents a simple and yet sufficiently realistic model [2] which gives a microscopic description of the Peierls distortion, and makes unambiguous predictions about the low-energy behavior. The previous paper looks carefully at the small polarons created by doping or photoemission. These were noticed already by $\mathrm{Yu}$, Chen, and $\mathrm{Su}$ [3]. Here we examine what happens when an electron-hole pair is inserted, and discover a self-trapped exciton which has not previously been discussed. This excitation gives a natural explanation for the Urbach tail seen in optical absorption [4] and for the multi-phonon replicas of the oxygen breathing phonon (the Peierls mode) seen by Kozlov et al. [4] in infrared absorption, and by Tajima et al. [5] in Raman scattering.

The model [1], based on work by Rice and Sneddon [2], has nearest-neighbor hopping (with strength $t$ ) of electrons between $\mathrm{Bi} s$-orbitals on a cubic lattice. Oxygens which lie between pairs of $\mathrm{Bi}$ atoms, experience a linear repulsive force $g\left(n_{2}-n_{1}\right)$ away from the $\mathrm{Bi}$ atom with the larger electron charge $\left(n_{1}\right)$ and toward the Bi atom with the smaller electron charge $\left(n_{2}\right)$. The oxygen atom also has a harmonic Einstein spring constant $K$. The model has a single dimensionless constant $\Gamma=g^{2} / K t$ in adiabatic approximation (motion of the oxygen atoms neglected.) We choose parameters $t=0.35 \mathrm{eV}, K=19$ $\mathrm{eV} / \stackrel{\circ}{A}^{2}, g=1.39 \mathrm{eV} / \stackrel{\circ}{A}$, yielding $\Gamma=0.30$.

\section{ATOMIC LIMIT $T=0$}

As in the previous paper, the zero bandwidth $(t=0$, $\Gamma \rightarrow \infty)$ case provides a soluble limit which gives good insight into the more complete theory. If a hole is created in the lower Peierls or occupied valence band, and an electron in the upper Peierls or empty conduction band, without allowing any lattice relaxation, the energy cost is $2 \Delta=24 g^{2} / K$. The hole is a $\mathrm{Bi}^{4+}$ ion on a site normally occupied by $\mathrm{Bi}^{3+}$, and the electron is a $\mathrm{Bi}^{4+}$ ion on a site normally occupied by $\mathrm{Bi}^{5+}$.

Because of the simple Hamiltonian, the problem has particle-hole symmetry. This is true at all values of parameters, not just $t=0$. Upper and lower Peierls bands have energies $\pm \lambda(\vec{k})$ where $\lambda(\vec{k})=\sqrt{\Delta^{2}+\epsilon(\vec{k})^{2}}$, and $\epsilon(\vec{k})$ is the nearest neighbor "cosine" band dispersion. Thus particle and hole bands are symmetrical.

If the electron and hole are spatially separated, each will lower its energy by lattice relaxation. Both electron and hole save $3 \Delta / 4$ in elastic energy by moving surrounding oxygens half way back to their cubic perovskite positions. However, each unpaired electron on a $\mathrm{Bi}^{4+}$ ion has its on-site energy increased by $\Delta / 2$, for a net lowering of energy by $\Delta / 4$ for each, or a cost of $3 \Delta / 2$ to create the isolated pair of polarons.

As shown in Fig. 1, there is an advantage to having the electron and hole polarons form on adjacent $\mathrm{Bi}$ ions. The oxygen between the two $\mathrm{Bi}^{4+}$ ions can further relax to the undistorted perovskite position. Rather than 12 , now only 10 oxygens are half displaced, saving an additional $\Delta / 12=g^{2} / K$ in elastic energy. This bound pair of polarons, called a "self-trapped exciton," costs $E_{\text {exc }}=17 \Delta / 12$ rather than $2 \Delta$ to create. The total energy gain from lattice relaxation is the exciton binding energy $\epsilon_{\text {exc }}=7 g^{2} / K$. Taking the on-site Coulomb interaction into account, the break-up of the $\mathrm{Bi}^{3+}$ paired electrons into separated electron-hole pairs will reduce the excited state energy further, to $\epsilon_{\mathrm{exc}}=7 g^{2} / K+U$ where $U$ is the Hubbard on-site Coulomb repulsion. 


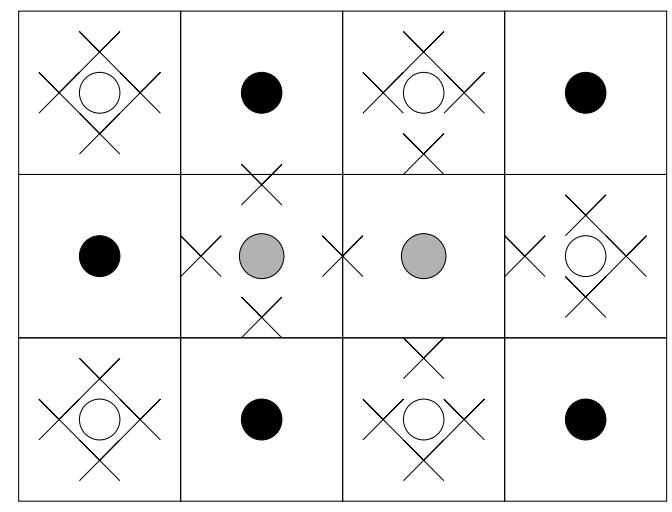

FIG. 1. Schematic structure of the self-trapped exciton. The electron is in the left-central cell ("exciton-electron"), the hole ("exciton-hole") in the right-central cell. Filled circles are $\mathrm{Bi}^{3+}$ ions with two $s$ electrons, shaded circles are $\mathrm{Bi}^{4+}$ ions with one $s$ electron, and open circles are $\mathrm{Bi}^{5+}$ ions with no $s$ electrons. X's denote oxygen ions. The central oxygen ion is undisplaced, which lowers the energy relative to separated electron and hole polarons.

The energy of the ground and excited state depend on the amount of lattice distortion. The configuration coordinate $\alpha$ smoothly connects the optimal oxygen configurations of the ground state at $\alpha=0$ and the self-trapped exciton state at $\alpha=1$, as shown in Fig. 2. The energy depends quadratically on $\alpha$, separating ground and excited state by the gap $2 \Delta$ at $\alpha=0$.

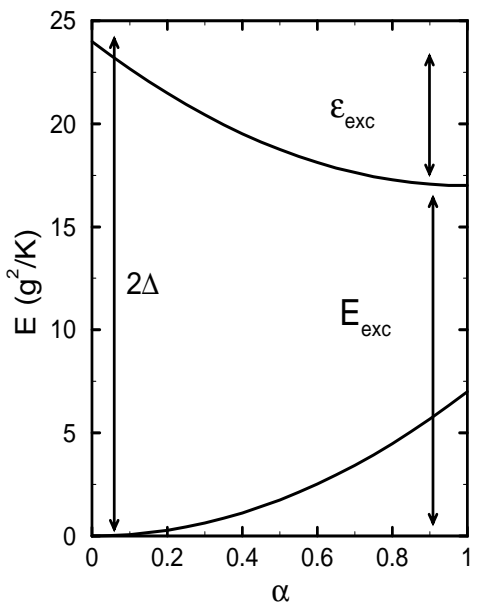

FIG. 2. Excited state and ground state energy versus configuration coordinate $\alpha$ for $t=0$. At $\alpha=0$, the ground and excited state are separated by the gap $2 \Delta$. The self-trapped exciton at $\alpha=1$ has its energy lowered by the maximal amount - the exciton binding energy $\epsilon_{\text {exc }}$. Including on-site Coulomb repulsion, the energy curve of the excited state will be shifted down by the Hubbard $U$.

\section{NUMERICAL RESULTS}

For $t \neq 0$ we are not able to solve analytically, and rely on numerical studies. We find that the excited state has zero slope $d E / d \alpha=0$ at $\alpha=0$, and downward curvature. No potential barrier separates the excited state at $\alpha=0$ and the self-trapped exciton at $\alpha=1$. This is contrary to most self-trapped exciton theories [6], where a metastable free excited state and a self-trapped exciton state can coexist, separated by a potential barrier.

For $t \neq 0$, localizing and delocalizing terms in the Hamiltonian compete against each other, yielding a critical coupling constant $\Gamma_{c}$ (exc) above which self-trapped exciton states exist. To obtain the lowest excited state numerically, oxygen positions are varied using the algorithm described in the preceding paper, under the constraint that an electron was removed from the highestlying valence band state and inserted in the lowest-lying conduction band state. We find $\Gamma_{c}(\mathrm{exc}) \approx 0.175$, close to the polaron stability limit $\Gamma_{c}(P)=0.18$ of the previous paper. Since the self-trapped exciton is an electronpolaron weakly bound to a hole-polaron, it is not surprising that the polaron and the self-trapped exciton have similar threshold coupling constants. The first correction to strong coupling for $\epsilon_{\text {exc }}$ can be approximated using a "vacuum correction" of order $1 / \Gamma^{2}$ discussed in Sect.III.A of the preceding paper: $\epsilon_{\mathrm{exc}} / t \approx 7 \Gamma-1 / 2 \Gamma$ and approaches asymptotically the $t=0$ limit $\epsilon_{\mathrm{exc}}=7 g^{2} / K$.

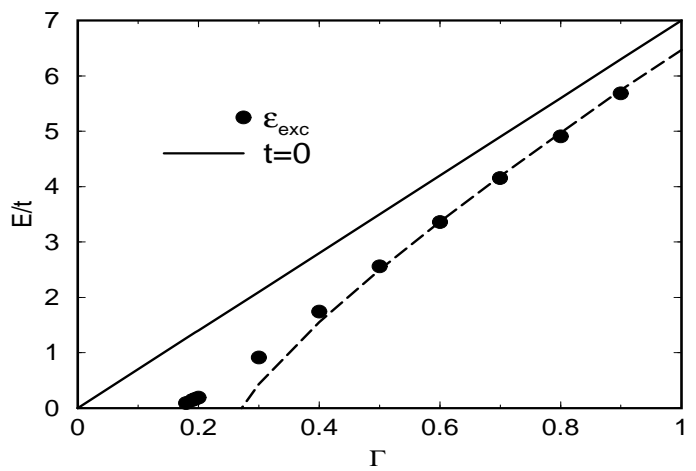

FIG. 3. Exciton binding energy $\epsilon_{\text {exc }}$ for $t \neq 0$ computed numerically. The bold solid line is the $t=0$ prediction. The dashed line is an approximate $1 / \Gamma^{2}$ correction.

The behavior of self-trapped exciton states and their evolution with $\Gamma$ is very similar that of polarons [1]. The local change in the oxygen environment leads to the appearance of localized gap states (Fig.(1) in addition to the states which constitute the exciton. 


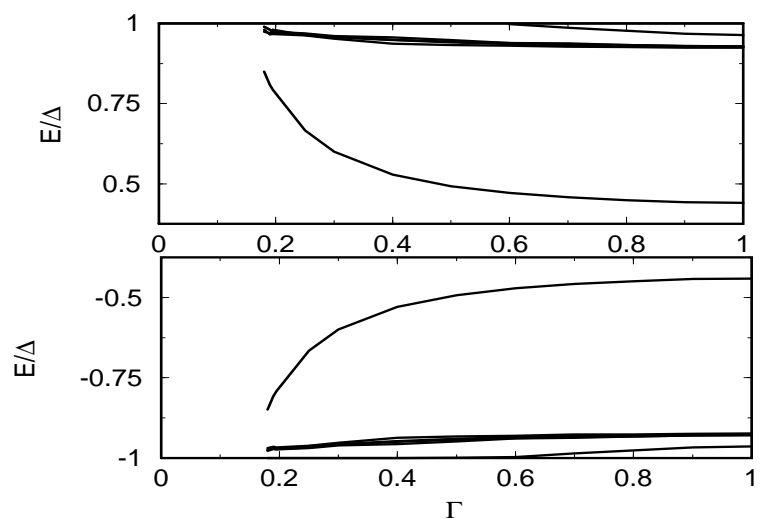

FIG. 4. Energies of localized single particle states which accompany the self-trapped exciton states lying in the gap. The spectrum has particle-hole symmetry. Five doubly occupied states split from the valence band. corresponding to states surrounding the exciton-electron. The mid-gap states derived from the valence and conduction band respectively are occupied singly each, corresponding to the exciton-hole and exciton-electron. Five empty states split from the conduction band. The ten states near the conduction and valence band edges are localized on the $10 \mathrm{Bi}$ atoms which surround the two $\mathrm{Bi}$ atoms which constitute the exciton.

Six states emerge from the valence band and six from the conduction band, symmetrically around $E=0$, because of electron-hole symmetry. The lowest five states have a small upward shift $\left(g^{2} / K\right.$ when $\left.t=0\right)$ from the top of the valence band, and remain doubly occupied. These are electrons on the five A sites surrounding the B sublattice site now occupied by the excited electron (called the "exciton-electron"). Their degeneracy is partly lifted when they couple to other sites by hopping, shifting their energies toward the valence band. The next state up also derives from the valence band, and is occupied once. It is the electron that remains behind on an A site when one electron is removed. Alternately, this is the site of the "exciton-hole". Its energy $\left(7 g^{2} / K\right.$ above the valence band when $t=0)$ is slightly higher than for an isolated hole state $\left(6 g^{2} / K\right)$ because one of the six surrounding oxygens, shared by the exciton-electron and the exciton-hole, is fully relaxed to the symmetric position. With decreasing coupling strength, the states spread out, as can be seen in the continuous evolution of their inverse participation ratios (IPR) in the inset in Fig. 5. The IPR $1 / P_{i}$ for a state $i$ is defined as

$$
\frac{1}{P_{i}}=\sum_{l}\left|\Psi_{i}\left(r_{l}\right)\right|^{4}
$$

where the sum runs over all sites. It measures the localization of a state. An IPR of $1 / P$ represents a state that is localized on $P$ atoms. The mean radius $\langle r\rangle$ of the two states comprising the exciton is shown in Fig. 5 . The particles are confined approximately to one site for $\Gamma>0.4$. For $\Gamma<0.4$, they delocalize rapidly, as indicated by the diverging radius. The self-trapped exciton evolves smoothly into a localized amplitude excitation of the charge-density wave, before disappearing into a band electron-hole pair at $\Gamma_{c}(E)$, just as [1] the small polaron evolves through a large CDW-like polaron state before becoming a band hole at $\Gamma_{c}(P)$.

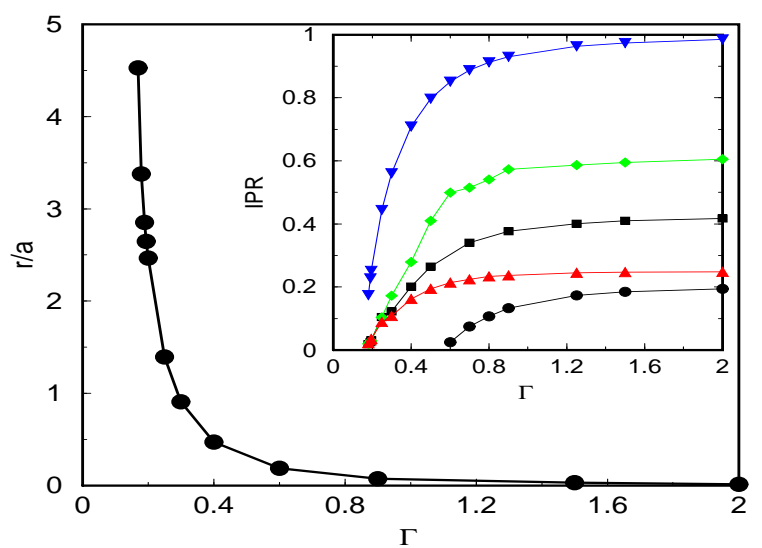

FIG. 5. Radius $\langle r\rangle$ of the exciton-hole and exciton-electron. A radius of 0 corresponds to complete localization of the wave-function at one site. The radius diverges as $\Gamma \rightarrow \Gamma_{c}(E)$. The finite value at $\Gamma_{c}(E)$ is caused by the finite system size of the numerical calculations. Inset: IPR-values of the gap states, indicating their degree of localization. They approach their asymptotic (symmetry determined) IPR values of $\frac{1}{5}, \frac{1}{4}$, $\frac{1}{2}$ (doubly degenerate), $\frac{13}{20}$ and 1 for $\Gamma \rightarrow \infty$.

\section{OPTICAL PROPERTIES}

In this section we discuss the observability of selftrapped excitons in experiment via an Urbach tail in optical absorption experiments and a multi-phonon spectrum of the "breathing mode" in phonon experiments, both governed by the Franck-Condon effect. Zheng and Nasu [7] have also obtained theoretically a tail in the optical conductivity. Their theory includes quantum lattice fluctuations (as does ours) and bears some relation to our theory.

As a first step we neglect both Coulomb and lattice polarization effects. Then the imaginary part of the dielectric constant can be computed exactly in electric dipole approximation. The dipole matrix element between an $s$ state on a $\mathrm{Bi}$ atom at $\vec{R}$ and one on the neighboring $\mathrm{Bi}$ atom at $\vec{R}+a \hat{x}$ is $-i m t a \hat{x} / \hbar$. This gives the correct intraband $\langle\vec{k}|\vec{p}| \vec{k}\rangle=(m / \hbar) \partial \epsilon / \partial \vec{k}$ relation between momentum matrix element and group velocity. Then for for unpolarized light we get

$$
\begin{aligned}
\epsilon_{2}(\omega) & =\frac{4 e^{2} t^{2}}{m^{2} \omega^{2} \pi \hbar^{2}} \int_{B Z} d^{3} \vec{k}\left(\sin ^{2}\left(k_{x} a\right)+\sin ^{2}\left(k_{y} a\right)+\sin ^{2}\left(k_{z} a\right)\right) \\
& \times \delta\left(2 \sqrt{\epsilon_{k}^{2}+\Delta^{2}}-\hbar \omega\right)
\end{aligned}
$$

This was evaluated numerically using a tetrahedron code [8.9], and is shown as the dashed line in Fig.6. The behavior of $\epsilon_{2}$ is dominated by the divergence of the 
joint-density of states at photon energy $\hbar \omega=2 \Delta$, corresponding to transitions from states at the top of the lower Peierls band into those at the bottom of the upper band. These states are mainly responsible for the charge disproportion and have vanishing amplitude on the $B$ and $A$ sites respectively. These are also the states from which localized polaron and self-trapped exciton states form. Since transitions between these states are highest in optical density, we may expect the line shape to be dominated by the signature of self-trapped excitons.

Materials with self-trapped excitons show FranckCondon broadening of electronic transitions, a concept well known to molecular spectroscopists, since molecular excited electronic states generally have altered nuclear coordinates. The process involves simultaneous creation of electronic excitation and nuclear vibrations, causing the appearance of vibrational sidebands in the electronic spectra 10]. In simple solids, excitations are usually delocalized which eliminates this effect [11]. However, in case of self-localized states, because of their significant lattice relaxation, Franck-Condon broadening of the electronic transitions must reappear. $\epsilon_{2}(\omega)$ in Franck-Condon approximation is given by:

$\epsilon_{2}(\omega) \propto\left|P_{\lambda \lambda^{\prime}}\right|^{2} \sum_{n} \sum_{n^{\prime}} w_{\lambda n}\left|\left\langle\lambda n \mid \lambda^{\prime} n^{\prime}\right\rangle\right|^{2} \delta\left(\hbar \omega+E_{\lambda n}-E_{\lambda^{\prime} n^{\prime}}\right)$,

where $P_{\lambda \lambda^{\prime}}$ is the electronic dipole matrix element between the electronic ground $\lambda$ and excited state $\lambda^{\prime}, w_{\lambda n}$ the probability to find $n$ vibrational quanta in the electronic ground state and $\left\langle\lambda n \mid \lambda^{\prime} n^{\prime}\right\rangle$ the overlap integral between the vibrational wave functions $\chi_{\lambda n}$. This integral can be large even when the vibrational quantum numbers $n, n^{\prime}$ are quite different from each other, because the vibrational wave-functions $\chi_{\lambda n}, \chi_{\lambda^{\prime} n^{\prime}}$ have origins which are off-set from each other.

At zero temperature, the broadening of the electronic transition derives from the overlap of zero point motion of the oxygens with excited vibrations in the electronic excited state. The sum over $n$ in Eq.(3) then has only one term, $w_{\lambda n}=\delta_{n, 0}$. We lift the adiabatic approximation applied so far by adding quantized vibrations, but treat the problem in the atomic limit $(t=0)$, i.e. in single site approximation. The excited electron states couple to eleven oxygens, which are treated as independent Einstein oscillators with frequency $\omega_{0}$ and oxygen mass $M$ in our model. They move in harmonic potentials and thus the Franck-Condon factor 12 is a product of eleven vibrational overlap integrals of the form $|\langle 0 \mid n\rangle|^{2}$ with:

$$
\langle 0 \mid n\rangle=\left(\frac{\alpha^{2}}{2}\right)^{\frac{n}{2}} \frac{\left(R-R^{\prime}\right)^{n}}{\sqrt{n !}} \exp \left(-\frac{\alpha^{2}}{4}\left(R-R^{\prime}\right)^{2}\right)
$$

where $\alpha=\sqrt{M \omega_{0} / \hbar}$ and $R$ and $R^{\prime}$ are the oxygen equilibrium positions of the ground and excited state respectively. The total absorption at a frequency $\hbar \omega=$ $E_{\text {exc }}+n \hbar \omega_{0}$, requires a sum over all different ways to distribute $n$ quanta over the eleven oxygens. The result is

$$
\begin{aligned}
\epsilon_{2}(\omega) & =\frac{4 \pi^{2} e^{2}}{m^{2} \omega^{2}} \sum_{n} \frac{1}{n !}\left(\frac{7 \Delta}{12 \hbar \omega_{0}}\right)^{n} \exp \left(-\frac{7 \Delta}{12 \hbar \omega_{0}}\right) \\
& \times \delta\left(\hbar \omega-\frac{17}{12} \Delta+n \hbar \omega_{0}\right) .
\end{aligned}
$$

In our simplified Einstein model, absorption occurs at discrete frequencies corresponding to different numbers of vibrations created in the absorption process. In reality these peaks are broadened by phonon dispersion, and discreteness of the individual peaks should only be seen for one or two vibrational quanta. The line shape should look similar to the envelope function of Fig.6, with maximal absorption at $\omega=2 \Delta$ and significant absorption below the gap at $2 \Delta=2 \mathrm{eV}$.

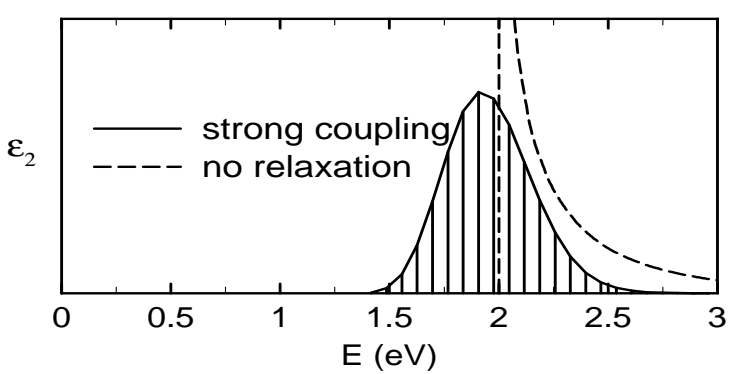

FIG. 6. Imaginary part of dielectric constant versus frequency. The dashed line shows Eq.(2), where the integral was evaluated with a tetrahedron code [8,9]. Taking lattice relaxation into account the absorption spectra will be dominated by the Franck-Condon effect Eq.(3), leading to significant peak broadening and absorption in the gap, known as an Urbach tail.

The absorption spectra of self-trapped excitons will change with temperature due to the temperaturedependent occupation of vibrational states in the electronic ground state given by $w_{\lambda, n}=\exp \left(\beta n \hbar \omega_{0}\right) / Z$, where the partition function $Z$ is $\left(1-\exp \left(\beta \hbar \omega_{0}\right)\right)^{-11}$ and $\beta=1 / k_{B} T$. Temperature enhances the absorption in the low energy regime and shifts the on-set of absorption to smaller energies. The temperature effect up to room temperature is very weak. At $T=300 \mathrm{~K}$ the probability to find a phonon on one of the eleven oxygens is less than $6 \%$. The analytical calculations for temperature corrections become increasingly tedious and Fig.7 shows a first temperature correction obtained numerically. 


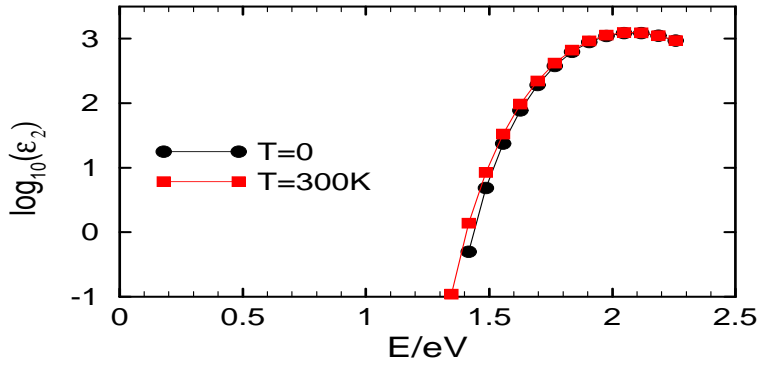

FIG. 7. Imaginary part of dielectric constant (logarithmic scale) versus frequency at $T=0$, and the first temperature correction to the Franck-Condon spectra at 300K.

The clearest evidence for self-trapped excitons is normally [6] observation of weak sub-gap features in optical absorption $\alpha(\omega) \propto \epsilon_{2}(\omega)$ and a large Stokes shift in the luminescence spectrum. We are not aware of luminescence measurements. Transient absorption measurements 13] have been interpreted as indicating that interband excitations relax rapidly to a state shifted down by $0.7 \mathrm{eV}$. Transmission measurements through thin single crystals were reported by Kozlov et al. [1]. These have been interpreted as showing an indirect band gap at 0.5 $\mathrm{eV}$ with anomalous temperature dependence. We believe that the measured absorption can be re-interpreted as an Urbach tail arising from the self-trapped exciton, with binding energy $\epsilon_{\text {exc }}$ enhanced beyond our values by the on-site Coulomb $U$.

Clear experimental support for the existence of selftrapped excitons in $\mathrm{BaBiO}_{3}$ comes from harmonics of the Peierls breathing mode phonon seen both in Raman experiments [5] and in the infrared [4]. These spectra are dominated by a strong peak at $570 \mathrm{~cm}^{-1}$, assigned to the breathing mode $Q=0 A_{1 g}$ phonon. Unlike in ordinary semi-conductors, a series of higher harmonics is observed. The simplest explanation for the Raman process is that the incoming photon creates a virtual self-trapped exciton, except that because of the Franck-Condon principle, the lattice coordinates stay at their ground state rather than their excited state values, which means that a cloud of virtual local vibrations is also created. When this virtual excitation re-radiates the scattered photon, not all the virtual vibrational quanta need to disappear. The test of this mechanism is that this process is resonant when the laser photon energy coincides with the Peierls gap. This resonance was seen by Tajima et al. [5]. Similar behavior occurs in the Raman spectrum of $\mathrm{LaMnO}_{3}$ [14] where a somewhat different species of selftrapped exciton is predicted to occur 15. In the infrared spectrum of $\mathrm{BaBiO}_{3}$, the selection rule against Raman phonons appearing in infrared must be violated by local loss of inversion symmetry due to static or dynamic lattice fluctuations. Multiples of the breathing phonon are as easily excited as a single phonon because of the vibrational overlap factors.

\section{ACKNOWLEDGMENTS}

We thank V. Perebeinos, V. Kostur, and R. Bhargava for help and encouragement. This work was supported by NSF grant no. DMR-0089492.

* Present address: Max-Planck-Institut für Kolloid- und Grenzflächenforschung, D-14424 Potsdam, Germany.

[1] I. Bischofs, V. Kostur, and P. B. Allen, preceeding paper.

[2] T. M. Rice and L. Sneddon, Phys. Rev. Letters 47, 689 (1982); P. Prelovsek, T. M. Rice, and F. C. Zhang, J. Phys. C 20, L229 (1987).

[3] J. Yu, X.-Y. Chen, and W. P. Su, Phys. Rev. B 41, 344 (1990).

[4] M. E. Kozlov, X. Ji, H. Minami, and H. Uwe, Phys. Rev. B 56, 12211 (1997).

[5] S. Tajima, S. Uchida, A. Masaki, H. Takagi, K. Kitazawa, S. Tanaka, and S. Sugai, Phys. Rev. B 35, 696 (1987).

[6] K. S. Song and R. T. Williams, Self-Trapped Excitons, 2nd Ed., (Springer, Berlin, 1996).

[7] H. Zheng and K. Nasu, Physica B 292, 344 (2000).

[8] G. Lehmann and M. Taut, Phys. Stat. Sol. (b) 54, 469 (1972); 57, 815 (1973).

[9] O. Jepsen and O. K. Andersen, Sol. State Commun. 9, 1763 (1971).

[10] G.Herzberg, Spectra of Diatomic Molecules, (Van Nostrand, 1950).

[11] E.I. Rashba, Opt. Spektrosk. 2, p.75 (1957).

[12] K. Huang and A. Rhys, Proc. R. Soc (London), Ser. A 204, 406 (1950).

[13] J. F. Federici, B. I. Greene, E. H. Hartford, and E. S. Hellman, Phys. Rev. B 42, 923 (1990).

[14] V. Perebeinos and P. B. Allen, cond-mat/0007301 and Phys. Rev. B (in press).

[15] P. B. Allen and V. Perebeinos, Phys. Rev. Letters 83, 4828-31 (1999). 